\title{
Differential Equations on Graphs *
}

\author{
A.I Vol'pert
}

\begin{abstract}
The differential equations encountered in various applications may be treated as equations on graphs. In the paper it is shown that the structure of the graph allows us to investigate the properties of the solutions of such equations.
\end{abstract}

Keywords and phrases: differential equations, graphs of reactions

Mathematics Subject Classification: 05C99, 34C99, 80A30, 92A05, 35K5O (1970)

\section{Introduction}

1.1. In the present paper we define differential equations on graph and we study certain properties of the solutions. It can be shown that the differential equations encountered in very different applications (chemical kinetics, chemical engineering, biology, markov processes, etc.) may be treated as equations on graphs. Difference schemes for certain classes of partial differential equations also are equations on graphs (section 5).

Thus, on the one hand, a sufficiently broad range of applications of the theory of such equations is possible, and, on the other hand, there is every reason to believe that the properties of the graph may be used to construct a qualitative theory of such equations and the methods for solving them.

On the example of the elementary properties it is shown here that a graph can be used to study the behaviour of the solutions of differential equations; for example, whether a solution is strictly positive or identically zero depends on whether we can go up to the corresponding vertex of the graph from the initial vertices along a path (section 2); the order of the zero of the solution depends upon the 'distance' of the given vertex from the initial vertices (section 3); the a priori estimate and the boundedness of the solution depend on the structure of the graph (section 4). Although in this article we consider only the elementary properties, there is reason to believe that the graph's structure influences the finer properties of the solution: stability, periodicity, asymptotics with respect to the coefficients, computation of the Ljapunov indices, etc. We remark also that here we are dealing with discrete graphs; however, the transition from difference equations to partial differential equations yields the possibility of interpreting the latter as equations on continuous graphs considered as the limiting case of discrete ones.

1.2. Here we shall define a system of differential equations on a graph. Let there be given a finite oriented bipartitie graph $(\Gamma)$ (for example, see [1]). This signifies that we are given two nonintersecting nonempty finite sets of objects:

$$
A=\left\{a_{i}\right\} \quad(i=1,2, \ldots, n), \quad B=\left\{b_{i}\right\} \quad(i=1,2, \ldots, m)
$$

*Reprinted with permission from Math. USSR Sbornik, Vol. 17 (1972), no. 4. 
(these points are called the vertices of the graph) and a certain finite set of ordered pairs of vertices (these pairs are called the edges of the graph), and, moreover, each pair contains vertices from the different sets indicated.

Let $\alpha_{i k}$ be the number of edges $\left\{a_{k}, b_{i}\right\}$, let $\beta_{i k}$ be the number of edges $\left\{b_{i}, a_{k}\right\}$. and let $\gamma_{i k}=\beta_{i k}-\alpha_{i k}$. we set $u=\left(u_{1}, \ldots, u_{n}\right)$ and we associate with each vertex $b_{i}$ a function $f_{i}(t, u)$ of $n+1$ variables, and with each vertex $a_{k}$, the function $u_{k}(t)$ and $g_{k}(t)$, where $f_{i}$ and $g_{k}$ are assumed as given and $u_{k}$ as being unknown.

Definition 1.1. The system

$$
\frac{d u_{k}}{d t}=\sum_{i=1}^{m} \gamma i k f_{i}(t, u)+g_{k}(t) \quad(k=1,2, \ldots, n)
$$

is called a system of differential equations on $\Gamma$.

We shall study the solutions of system (1) for $t>0$ satisfying the initial conditions

$$
u_{k}(0)=u_{k}^{0} \quad(k=1,2, \ldots, n),
$$

and, moreover, we shall assume that

$$
u_{k}^{0} \geq 0 \quad(k=1,2, \ldots, n) .
$$

The functions $f_{i}(t, u)$ are assumed given in the halfspace $t \geq 0$, continuous in $t$ and $u$, and continuously differentiable in $u_{k}(k=1, \ldots, n)$. We assume also that

$$
\left.f_{i}(t, u) \geq 0\right)
$$

for $t>0$ and $u_{k} \geq 0 \quad(k=1, \ldots, n)$.

The function $g_{k}(t)$ are assumed given for $t \geq 0$, continuous (for simplicity), and nonnegative.

1.3. The graph $(\Gamma)$ can be specified as the mapping of linear forms

$$
\sum_{k=1}^{n} \alpha_{i k} \alpha_{k} \rightarrow \sum_{k=1}^{n} \beta_{i k} \alpha_{k} \quad(i=1,2, \ldots, m)
$$

with nonnegative integral coefficients. The $a_{k}(k=1, \ldots, n)$ are the $A$-vertices of graph $\Gamma$, and the mappings (5) are the $B$-vertices. The numbers $\alpha_{i k}$ and $\beta_{i k}$ denote the numbers of corresponding edges. The system of mappings (5) will be called the scheme of the graph $\Gamma$.

If we are given a graph $\Gamma$ with its scheme (5) and a graph $\Gamma^{\prime}$ with its scheme

$$
\sum_{l=1}^{n^{\prime}} \alpha_{i l}^{\prime} \alpha_{l}^{\prime} \rightarrow \sum_{l=1}^{n^{\prime}} \beta_{i l}^{\prime} \alpha_{l}^{\prime} \quad\left(i=1,2, \ldots, m^{\prime}\right),
$$

then by $\Gamma \times \Gamma^{\prime}$ we shall denote the graph whose $A$-vertices are the pairs $a_{k l}=\left(a_{k}, a_{l}^{\prime}\right)$ with the scheme

$$
\begin{array}{ll}
\sum_{k=1}^{n} \alpha^{i k} \alpha^{k l} \rightarrow \sum_{k=1}^{n} \beta_{i k} \alpha_{k l} & \left(i=1,2, \ldots, m ; \quad l=1,2, \ldots, n^{\prime}\right), \\
\sum_{l=1}^{n^{\prime}} \alpha_{i l}^{\prime} \alpha_{k l} \rightarrow \sum_{l=1}^{n^{\prime}} \beta_{i l}^{\prime} \alpha_{k l} & \left(i=1,2, \ldots, m^{\prime} ; \quad k=1,2, \ldots, n\right) .
\end{array}
$$


Thus the graph $\Gamma \times \Gamma^{\prime}$ contains $n n^{\prime} A$-vertices and $m n^{\prime}+m^{\prime} n B$-vertices.

1.4. As an example let us consider an equation of chemical kinetics. Suppose we are given a system of reactions in which the substances $a_{k}(k=1, \ldots, n)$ take part:

$$
\sum_{k=1}^{n} \alpha_{i k} \alpha_{k} \rightarrow \sum_{k=1}^{n} \beta_{i k} \alpha_{k} \quad(i=1,2, \ldots, m),
$$

where $\alpha_{i k}$ and $\beta_{i k}$ are the stoichiometric coefficients. Let $f_{i}(t, u)$ be the rate of the $i t h$ reaction, being a function of time $t$ and of the concentrations $u_{k}$ of the substances $a_{k}(k=1, \ldots, n)$. Then the system of equations of chemical kinetics, corresponding to the system (6) of reactions, takes the form (1) (with $\left.g_{k}=0\right)$ (see [2]). If we now treat (6) as the scheme of a graph $\Gamma$, then the equations of chemical kinetics are equations on $\Gamma$. Since the reaction rate is a nonnegative quantity, condition (4) is fulfilled. Usually the functions

$$
f_{i}(t, u)=k_{i} u_{1}^{\alpha_{i 1}} u_{2}^{\alpha_{i 2}} \ldots u_{n}^{\alpha_{i n}},
$$

where $k_{i}$ are given positive constants (constants of the reaction rates), are chosen as the $f_{i}(t, u)$. Here, in (7) we have assumed $u_{l}^{\alpha_{i l}}=1$ for $a_{i l}=0, u_{l} \neq 0(l=1, \ldots, n)$.

If the diffusion processes are examined simultaneously with the kinetics, then we obtain a system of partial differential equations and, moreover, the corresponding system of difference (in the space variables) equations is a system of equations on $\Gamma \times \Gamma_{1}$, where $\Gamma$ describes, the chemical kinetics process and $\Gamma_{1}$, the diffusion (section 5).

\section{Positiveness of the solution}

Here we shall show that under specific natural conditions on the functions $f_{i}(t, u)$, for nonnegative $g_{k}$ and initial conditions, the solution of system (1) is nonnegative. Furthermore, the solutions are strictly positive at all vertices of graph $\Gamma$ which are connected with the initial vertices by paths in $\Gamma$ (such vertices are called reachable). It turns out, further, that for $g_{k}=0$ the solution is identically zero at the unreachable vertices.

2.1. Here we prove the nonnegativeness of the solution. A vertex $a_{k}\left(b_{i}\right)$ is said to be directly preceding to the vertex $b_{i}\left(a_{k}\right)$ if $\alpha_{i k}>0\left(\beta_{i k}>0\right)$. In what follows it is assumed that every $B$-vertex has at least one directly preceding $A$-vertex. We shall say that a function $f_{i}(t, u)$ is subordinate to the vertex $a_{k}$, if $f_{i}(t, u)=0$ for $u_{k}=0$.

Condition I. The function $f_{i}(t, u)$ is subordinate to all $A$-vertices directly preceding to the vertex $b_{i}$.

This condition has a natural physical sense. For example, in chemical kinetics it signifies that the reaction rate equals zero if the concentration of the reactant equals zero.

Theorem 2.1. If $u_{k}^{0}>0, g_{k}(t) \geq 0(k=1, \ldots, n)$ and Condition $\boldsymbol{I}$ is fulfilled, then the smooth solution of problem (1), (2) is positive:

$$
u_{k}(t)>0 \quad(t \geq 0, k=1,2, \ldots, n)
$$

on the interval where this solution exists. 
Proof. Assume that the solution exists on the interval $[0, T]$ but that (8) does not hold. Then the solutions intersect the $t$-axis. Let $t_{0}$ be the leftmost point of intersection, so that

$$
U_{s}(t)>0 \quad\left(0 \leq t<t_{o}, s=1,2, \ldots, n\right) .
$$

For definiteness let

$$
u_{k}\left(t_{o}\right)=0
$$

In system (1) we consider those $i$ for which $\gamma_{i k}<0$. Then $\alpha_{i k}>0$, i.e. $a_{k}$ directly precedes $b_{i}$, and consequently $f_{i}(t, u)=0$ when $u_{k}=0$, so that

$$
f_{i}(t, u(t))=\varphi_{i}(t) u_{k}(t)
$$

We introduce the notation

$$
\varphi(t)=\sum^{\prime \prime} \gamma_{i k} \varphi_{i}(t)
$$

where the summation is carried out over all $i$ for which $\gamma_{i k}<0$, and

$$
\psi(t)=\sum_{i} \gamma_{i k} f_{i}(t, u(t))+g_{k}(t)
$$

where the summation is carried out over all $i$ for which $\gamma_{i k} \geq 0$. Obviously $\psi(t) \geq 0$ on the interval $\left[0, t_{0}\right]$. From (1) we now obtain

$$
u_{k}(t)=\varphi(t) u_{k}(t)+\psi(t)
$$

whence

$$
u_{k}(t)=u_{k}(0) \exp \left\{\int_{0}^{t} \varphi(s) d s\right\}+\int_{0}^{t} \exp \left\{\int_{r}^{t} \varphi(s) d s\right\} \psi(r) d r
$$

Consequently $u_{k}\left(t_{0}\right)>0$, which contradicts (9). The theorem is proved.

Theorem 2.2. If $u_{k}^{0} \geq 0, g_{k}(t) \geq 0(k=1, \ldots, n)$ and Condition $\boldsymbol{I}$ is fulfilled, then the smooth solution of problem (1), (2) is nonnegative:

$$
u_{k}(t) \geq 0 \quad(t \geq 0, k=1,2, \ldots, n)
$$

on the interval where this solution exists.

The proof follows from Theorem 2.1 and the well-known theorem on the continuous dependence of solutions on initial conditions (for example, see [3]), since it suffices to take $U_{k}^{0}+\epsilon(k=1, \ldots, n ; \epsilon>0)$ as the initial conditions and then to pass to the limit as $\epsilon \rightarrow 0$.

2.2. Here we define a $B$-path and a $B$-reachable vertex in the graph $\Gamma$. By an acyclic graph we mean a graph in which there are no oriented cycles ([1], Russian p. 37).

Definition 2.3. The acyclic part $S$ of a graph $\Gamma$, containing together with each $B$-vertex all the arriving edges and together with each edge both the incident vertices, is called a $B$-path.

We shall assume that the orientation of the edges of graph $\mathrm{G}$ is preserved in $\mathrm{S}$. An $A$-vertex is called initial in $\mathrm{S}$ if it does not have arriving edges in $\mathrm{S}$. 
Definition 2.4. Let there be given a set $\left(A_{0}\right)$ of $A$-vertices. An $A$-vertex $\left(a_{k}\right)$ is said to be $B$-reachable from $A_{0}$ if there exists a $B$-path containing $a_{k}$, all of whose initial vertices belong to $A_{0}$.

Since other concepts of paths and of reachability will not be used here, we shall simply speak of a "path" and of a "reachable vertex" instead of a " $B$-path" and a " $B$-reachable vertex."

In what follows we shall need the following indexing of the graph vertices. We take the set $A_{0}$ as the initial set and we ascribe the index 0 to all the vertices occurring in it. The index 0 is assigned also to those $B$-vertices all of whose directly preceding vertices have been given index 0 . Further indexing is carried out by induction. Suppose that indices up to $s$, inclusive, have already been ascribed to the A- and $B$-vertices. Then the index $s+1$ is ascribed to all the unindexed $A$-vertices for which there exist directly preceding $B$-vertices with index $s$. Next, the index $s+1$ is ascribed to all the unindexed $B$-vertices all of whose directly preceding $A$-vertices have an index.

Since the graph $\Gamma$ is finite, this process terminates after a finite number of steps. However, generally speaking, not all vertices of the graph will receive an index by this process. All vertices not provided with an index by this process will be assigned the index $+\infty$.

Theorem 2.5. In order for an A-vertex to have a finite index it is necessary and sufficient that it be reachable from $A_{0}$.

Proof. Necessity. Suppose that vertex $a_{k}$, has a finite index. We construct $S$ in the following manner. At the first step we include vertex $a_{k}$, in $S$. Suppose that $n$ steps have been taken. The continuation of the path takes place for the $A$-vertices included in $S$ at the $n t h$ step, not belonging to $A_{0}$ and not included in $S$ at the preceding steps. We denote these vertices by $\bar{a}_{k}(k=1, \ldots, r)$. For each vertex $\bar{a}_{k}$, we select one directly preceding $B$-vertex $\left(b_{k}\right)$ with a smaller index. Let $a_{k i}\left(i=1, \ldots, s_{k}\right)$ be all the $A$-vertices directly preceding $b_{k}$. Then at the $(n+1) t h$ step the vertices $b_{k}$, and $a_{i k}$, as well as the edges $\left(b_{k}, \bar{a}_{k}\right)$ and $\left(a_{k i}, b_{k}\right)\left(i=1, \ldots, s_{k} ; k=1, \ldots, r\right)$, are included in $S$. (Here different designations are possible for one and the same vertices and edges).

It is clear that the process terminates after a finite number of steps. From the construction method we conclude the following. All the vertices occurring in $S$ have a finite index. Further, for edges of the form $\{b, a\}$ occurring in $S$ the index of vertex $b$ is less than the index of vertex $a$, while for edges of the form $\{a, b\}$ the index of vertex $a$ does not exceed the index of vertex $b$. Therefore there cannot be oriented cycles in $S$. Finally, in $S$ there may not occur $A$-vertices not belonging to $A_{0}$ and not having in $\Gamma$ directly preceding $B$-vertices, because from the vertex indexing method it follows that such vertices have an infinite index. Hence it follows that all initial $A$-vertices in $S$ belong to $A_{0}$.

Thus $S$ is the desired path and the vertex $a_{k}$, is reachable from $A_{0}$.

Sufficiency. Let a be $a_{k}$ reachable vertex and let $S$ be the path occurring in the definition of reachability. Further, let $X$ be the set of vertices with an infinite index. Assume that $a_{k} \in X$. Then $a_{k} \notin A_{0}$ and, therefore, is not an initial vertex in $S$. Let $b_{i}$ be a directly preceding $B$-vertex, $b_{i} \in S$. Then $b_{i} \in X$. Among the $A$-vertices directly preceding $b_{i}$ (they all belong to $S$ ), we can find at least one from $X$. Continuing further in this way and taking into account the absence of cycles in $S$, we would obtain an unbounded number of vertices, which is impossible. The theorem is proved.

2.3. Here we shall investigate the question of the positiveness of the solution of system (1). By $A_{0}$ we denote the set of all those vertices $a_{k}$ for which $u_{k}^{0}>0$, so that $u_{k}^{0}=0$ at all the remaining $A$-vertices.

Theorem 2.6. In system (1) let $g_{k}=0(k=1, \ldots, n)$ and let Condition I be fulfilled. Further, let $u(t)$ be a solution of problem (1), (2) on the interval $[0, T]$. Then

$$
u_{k}(t) \equiv 0 \quad(0 \leq t \leq T)
$$

at all the vertices $a_{k}$ of the graph $\Gamma$ unreachable from $A_{0}$. 
Proof. By $X$ we denote the set of all unreachable vertices and by $N$ the set of numbers of all $A$-vertices from $X$. Let $k \in N$. Let us prove that if $\gamma_{i k} \neq 0$, then

$$
f_{i}(t, u(t))=\varphi_{i}(t) u_{l}(t)
$$

where $l \in N, \phi_{i}(t)$ is a continuous function on the interval $[0, T]$. Indeed, if $\alpha_{i k}>0$, then $f_{i}(t, u)=0$ when $u_{k}=0$, i.e $l=k$ in (14). If $\alpha_{i k}=0$ and $\beta_{i k}>0$, then among the $A$-vertices directly preceding $b_{i}$ there is at least one unreachable one. Therefore (14) holds.

In (1) we now consider only the equations for $u_{k}$ for all $k \in N$. On the basis of (14) we obtain a system of linear equations. Since $u_{k}^{0}=0(k \in N)$, this system has only the trivial solution. The theorem is proved.

We impose an additional condition on the functions $f_{i}$

Condition II. If $u_{k}>0$ at all vertices $a_{k}$ directly preceding to the vertex $b_{i}$, then $f_{i}(t, u)>0$ for $t \geq 0, u_{k}>0 \quad(k=1, \ldots, n)$.

This condition is fulfilled, in particular, for the equations of chemical kinetics (see (7)), as well as for parabolic difference equations (see section 5).

Theorem 2.7. Let Conditions I and II be fulfilled. Further, let u(t) be a solution of problem (1), (2) on the interval $[0, T]$. Then

$$
u_{k}(t)>0 \quad(0<t \leq T)
$$

for all vertices $a_{k}$ of the graph $\Gamma$ reachable from $A_{0}$.

Proof. We carry out an induction on the indices. By the definition of set $A_{0}$ we have $u_{k}^{0}>0$ for all $A$ vertices $\left(a_{k}\right)$ with index 0 . By repeating the calculations in the proof of Theorem 2.1 we obtain equality (12) for $t \in[0, T]$, and, moreover, $\psi(t) \geq 0$. Hence it follows that $u_{k}(t)>0$. Thus the theorem is true for vertices with index 0 .

Suppose that we have already proven that $u_{k}(t)>0$ for all vertices $a_{k}$, with an index less than or equal to $s$. Consider a vertex $a_{k}$, with index $s+1$. Then we can find at least one $B$-vertex, directly preceding $a_{k}$ with an index equal to $s$. Let this be $b_{l}$. Since all $A$-vertices directly preceding $b_{l}$ have an index not exceeding $s$, at them the solution is positive. By virtue of Condition II, $f_{l}(t, u)>0$. Thus $\psi(t)>0$ in equation (11) and, on the basis of $(12), u_{k}(t)>0$. The theorem is proved.

\section{Behaviour at zero}

As above, let $A_{0}$ be the set of all $A$-vertices of the graph $\Gamma$ at which the initial conditions are positive. Here we shall point out the connection between the distribution of the vertices relative to $A_{0}$ and the order of the zero of the solution of problem (1), (2).

We shall assume that $g_{k}(t)=0(k=1, \ldots, n)$ and that if $\kappa=\max \kappa_{l}$, where $\kappa_{l}$ is the index of vertex $a_{l}$ and the maximum is taken over all vertices reachable from $A_{0}$, then the function $f_{i}(t, u)$ have continuous partial derivatives up to order $\max \{\kappa-1,1\}$.

Theorem 3.1. Let Condition I be fulfilled, let $a_{l}$ be a vertex with index $\kappa_{l}$, reachable from $A_{0}$, and let $u(t)$ be a solution of problem (1), (2). Then, in a neighborhood of zero,

$$
u_{l}(t)=t^{\chi_{l}} v_{l}(t)
$$

where $v_{l}(t)$ is a continuous function. 
Proof. Equality (15) is trivial when $\kappa_{l}=0$. Let $\kappa_{l}>0$. We are required to prove that

$$
u_{l}^{(r)}(0)=0 \quad(r=1,2, \ldots, \chi-1)
$$

for all those $l$ for which $\kappa_{l}>r$. It is obvious that (15) follows from this.

We carry out an induction on $r$. When $r=0,(16)$ is trivial because if $\kappa_{l}>0$, then $a_{l} \notin A_{0}$, and consequently $u_{l}(0)=0$. Suppose that equality (16) is true for $r \leq s-1$. Let us prove it for $r=s$. Let $\kappa_{l}>s$. Then the indices of all the $B$-vertices directly preceding $a_{l}$ are not less than $s$. Thus in the equality

$$
u_{l}=\sum_{l} \gamma_{i l} f_{i}(t, u)
$$

all those $i$ for which $\gamma_{i l}>0$ are the numbers of the $B$-vertices with indices not less than s. Any such vertex $b_{i}$ has, among the directly preceding ones, at least one $A$-vertex $a_{k}$, with index not less than $s$. Since Condition I is fulfilled for system (1) by assumption,

$$
f_{i}(t, u(t))=\varphi_{i}(t) u_{k}(t) \quad\left(\chi_{k} \geq s\right) .
$$

If $\gamma_{i l}<0$, then $\alpha_{i l}>0$, and therefore $f_{i}(t, u)=\phi_{i}(t) u_{l}(t)$. Thus (17) can be written as

$$
\dot{u}_{l}=\sum_{i} \varphi_{l}(t) u_{k_{l}}
$$

moreover, $\kappa_{k_{i}} \geq s$. By the induction assumption $u_{k_{i}}^{(r)}(0)=0$ for all $r$ less than $s$. From this and from (18) it follows that $u_{l}^{(r)}(0)=0$ for all $r$ not exceeding $s$. The theorem is proved.

We remark that equality (15) holds formally for small $t>0$ and for the unreachable vertices (on the basis of Theorem 2.6).

In equality $(15), v_{l}(0)$ can be equal to zero, because the order of the zero of the function $u_{l}$, at zero can be greater than $\kappa_{l}$.

Theorem 3.2. If $f_{i}(t, u)(i=1, \ldots, m)$ are linear functions and if Conditions I and II are fulfilled, then the order of the zero of the solution $u_{l}(t)$ for each reachable vertex $a_{l}$ equals its index $\kappa_{l}$.

Proof. We are required to prove that $v_{l}(0) \neq 0$ in (15). From the linearity of the function $f_{i}(t, u)$ in $\mathrm{u}$ and from Condition I it follows that $f_{i}(t, u)=\phi_{i}(t) u_{k_{i}}$. From Condition II we obtain the inequality $\phi_{i}(t)>0$ for $t \geq 0$. On the basis of (17) we now have

$$
\dot{u}_{l}=\varphi(t) u_{l}+\sum_{i}{ }^{\prime} \gamma_{i l} \varphi_{i}(t) u_{k_{i}},
$$

where the prime denotes that the sum is taken over positive $\gamma_{i l}$.

We prove the inequality $v_{l}(0) \neq 0$ by induction on $\kappa_{l}$. It is trivial for $\kappa_{l}=0$. Suppose that it has already been proven for all $l$ with $\kappa_{l}<s$. Let $\kappa_{l}=s$. On the basis of Theorem $3.1, u_{l}=t^{s} v_{l}(t)$, and $u_{k_{i}}=t^{\kappa_{k_{i}}} v_{k_{i}}(t)$; moreover, $\kappa_{k_{i}} \geq s-1$ and

$$
\chi_{k_{i}}=s-1
$$

for at least one $i$. For this $i$, by the induction assumption, $v_{k_{i}}(0)>0$. All terms on the right in (19), for which (20) holds, occur with like sign, and therefore (19) can be written as $\dot{u}_{l}=c(t) t^{s-1}$, where $c(0)>0$. Hence it follows that $u_{l}(t)=v_{l}(t) t^{s}$ and $v_{l}(0)>0$. The theorem is proved. 


\section{A priori estimates}

Here we obtain a priori estimates for the solution of system (1), determinable from its graph and not depending on the functions $f_{i}(t, u)$. We assume that Condition I is fulfilled.

We consider the linear forms

$$
L_{i}(\lambda)=\sum_{k=1}^{n} \gamma_{i k} \lambda_{k} \quad(i=1,2, \ldots, m) .
$$

Lemma 4.1. Let $u(t)$ be a solution of problem (1), (2) with nonnegative initial conditions. If $\lambda_{1}, \ldots, \lambda_{n}$ is a solution of the system of inequalities

$$
L_{i}(\lambda) \leq 0 \quad(i=1,2, \ldots, m)
$$

then

$$
v(t)=\sum_{l=1}^{n} \lambda_{l}\left[u_{l}(t)-\int_{0}^{t} g_{t}(\tau) d \tau\right]
$$

is a nonincreasing function of $t$.

Proof. From (22) we have

$$
v(t)=\sum_{l=1}^{n} \lambda_{l}\left[\dot{u}_{l}(t)-g_{l}(t)\right]=\sum_{l=1}^{m} L_{l}(\lambda) f_{l}(t, u) \leq 0,
$$

because $f_{i}(t, u) \geq 0$, whence follows the lemma's assertion.

Obviously, if instead of (21) there occurs an inequality in the opposite direction, then $v(t)$ is a nondecreasing function. If the collection $\lambda_{1}, \ldots, \lambda_{n}$ is the solution of the system of equations

$$
L_{i}(\lambda)=0 \quad(i=1,2, \ldots, m),
$$

then $v(t)=$ const.

Theorem 4.2. Let there exist a nonnegative solution of system (21):

$$
\lambda_{l} \geq 0 \quad(l=1,2, \ldots, n)
$$

where $\lambda_{k} \geq 1$. Further, let $u(t)$ be a solution of problem (1), (2) with nonnegative initial conditions. Then

$$
0 \leq u_{k}(t) \leq \sum_{l=1}^{m} \lambda_{l}\left[u_{l}^{0}+\int_{0}^{t} g_{t}(\tau) d \tau\right]
$$

The proof follows from the lemma and from the inequality $v(t) \leq v(0)$.

Remark. The determination of the optimal a priori estimate on the interval $[0, T]$ for $u_{k}(t)$ is a linear programming problem: find

$$
\min _{\lambda} \sum_{l=1}^{m} \lambda_{l}\left[u_{l}^{0}+\int_{0}^{T} g_{l}(t) d t\right]
$$

in the region: $L_{i}(\lambda) \leq 0(i=1, \ldots, m), \lambda_{l}>0(l=1, \ldots, n ; l \neq k), \lambda_{k} \geq 1$. 
Theorem 4.3. (The existence theorem). If positive solutions $\lambda_{l}>0 \quad(l=1, \ldots, m)$ of inequalities (21) exist, then the solution of problem (1), (2) with nonnegative initial conditions exists on the semiaxis $t>0$.

Proof. From Theorem 4.2 follows an a priori estimate on any interval $[0, T]$, which obviously is sufficient for the existence of a solution.

Remark. The requirement of the existence of a positive solution of system (21) (and even of system (23)) has a physical meaning. For example, in chemical kinetics this denotes the presence of material equilibrium in the reaction system (6).

\section{Partial differential equations}

Here we present some examples of partial differential equations for which the corresponding difference equations (in the space variables) may be interpreted as equations on graphs with the fulfilment of Condition I.

5.1. First let us consider as an example the one-dimensional parabolic equation

$$
\frac{\partial u}{\partial t}=\frac{\partial}{\partial x}\left(a(t, x, u) \frac{\partial u}{\partial x}\right)
$$

on the segment $0 \leq x \leq 1$ with the initial and boundary conditions

$$
\left.u\right|_{t=0}=\varphi(x),\left.\quad u\right|_{x=0}=\psi_{0}(t),\left.\quad u\right|_{x=1}=\psi_{1}(t) .
$$

We assume that the function $a(t, x, u)>0$ has been given for $t \geq 0,0 \leq x \leq 1$, and for all $u$, and satisfies the condition

$$
a(t, x, u)>0
$$

for $t \geq 0,0 \leq x \leq 1, u \geq 0$.

We divide up the segment $[0,1]$ by the partition points $x_{0}, x_{1}, \ldots, x_{r}\left(x_{0}=0, x_{r}=1\right)$ into $r$ equal parts, and we let $h=1 / r$. We specify a graph by a scheme in the following way:

$$
\begin{array}{r}
x_{n} \rightarrow x_{n+1}, \\
x_{n+1} \rightarrow x_{n} \quad(n=0,1, \ldots, r-1) .
\end{array}
$$

With the $B$-vertex (26) there is associated the function $h^{-2} a\left(t, x_{n}, u_{n}\right) u_{n}$, and with the $B$-vertex $(27)$, the function $h^{-2} a\left(t, x_{n}, u_{n}\right) u_{n+1}$. Then the equation on the graph (26), (27) has the form

$$
\begin{gathered}
\frac{d u_{n}}{d t}=\frac{1}{h}\left[a\left(t, x_{n}, u_{n}\right) \frac{u_{n+1}-u_{n}}{h}-a\left(t, x_{n-1}, u_{n-1}\right) \frac{u_{n}-u_{n-1}}{h}\right] \\
(n=1,2, \ldots, r-1)
\end{gathered}
$$

and is obviously the difference approximation (with respect to $x$ ) of equation (24). Here the equations are not written for the vertices $x_{0}$ and $x_{r}$ and it is assumed that $u_{0}(t)=\psi_{0}(t)$ and $u_{r}(t)=\psi_{1}(t)$. Conditions 
I and II are clearly fulfilled in the case being considered, and therefore the conclusions drawn in section 2 and section 3 are valid for the solutions of system (28).

5.2. We now consider the first boundary value problem for the parabolic system of equations

$$
\begin{gathered}
\frac{\partial u_{k}}{\partial t}=\frac{\partial}{\partial x}\left(a_{k}\left(t, x, u_{k}\right) \frac{\partial u_{k}}{\partial x}\right)+\sum_{i=1}^{m} \gamma_{i k} f_{i}(t, x, u)+g_{k}(t, x) \\
\left(k=1,2, \ldots, n ; u=\left(u_{1}, u_{2}, \ldots, u_{n}\right)\right)
\end{gathered}
$$

on the segment $0 \leq x \leq 1$.

We assume that the graph $\Gamma$ is given by its scheme (5), $\gamma_{i k}=\beta_{i k}-\alpha_{i k}$ and that the $f_{i}(t, x, u)$ are functions given on the $B$-vertices of $\Gamma$ in just the same way as in section 1 with the sole difference that they are now functions of $t, x$ and $u$. Let $\Gamma_{1}$ be the graph mentioned in section 1 . From the preceding it is clear how to specify functions at the $B$-vertices of the graph $\Gamma \times \Gamma_{1}$ so as to obtain a difference approximation (relative to $x$ ) of the system (29) with the fulfillment of Condition I (if this condition is fulfilled on $\Gamma$ ).

Analogous constructions may be implemented for the first boundary value problem for the parabolic system

$$
\begin{gathered}
\frac{\partial u_{k}}{\partial t}=\sum_{l=1}^{N} \frac{\partial}{\partial x_{l}}\left(a_{k l}\left(t, x, u_{k}\right) \frac{\partial u_{k}}{\partial x_{l}}\right)+\sum_{i=1}^{m} \gamma_{i k} f_{i}(t, x, u)+g_{k}(x, t) \\
\left(k=1,2, \ldots, n, \quad u=\left(u_{1}, u_{2}, \ldots, u_{n}\right), \quad x=\left(x_{1}, x_{2}, \ldots, x_{N}\right)\right)
\end{gathered}
$$

Considered in a bounded region of an $N$-dimensional space.

The requirement (25) of strict parabolicity is not a necessary one for the analysis carried out. We can restrict ourselves by the condition $a(t, x, u) \geq 0$ and by analogous conditions for systems (29) and (30) with an appropriate formulation of the boundary conditions.

5.3. The number of examples of partial differential equations presented can be increased considerably. We note that if a derivative of the form $\partial b(t, x) u / \partial x$ occurs, then (in the one-dimensional case) there corresponds to it a graph with the scheme $x_{n} \rightarrow x_{n+1}$ or $x_{n+1} \rightarrow x_{n}$ depending on the sign of $b$. This makes it possible to insert terms of the indicated form (as well as of the form $\partial b(t, x, u) / \partial x$ ) into the systems being analyzed, and also to study certain classes of first-order hyperbolic systems, for example, systems of the form

$$
\frac{\partial u_{k}}{\partial t}=\sum_{l=1}^{N} \frac{\partial b_{k l}\left(t, x, u_{l}\right)}{\partial x_{t}}+\sum_{l=1}^{m} \gamma_{i k} f_{i}(t, x, u)+g_{k}(t, x),
$$

where the notation is the same as in (30).

\section{References}

[1] O. Ore. The theory of graphs. Amer. Math. Soc. Colloq. Publ., vol.38, Amer. Math. Soc., Providence, R.I., 1962; Russian transl., "Nauka", Moscow, 1968. MR 27 \# 740; 39 \# 88.

[2] N. M. Èmmanuèł', D.G. Knorre. Course of chemical kinetics (homogenous reactions), 2nd ed., Vysšaja Škola, Moscow, 1969. (Russian) CA 73 \# 29434n.

[3] V. V. Nemyckii, V.V. Stepanov, Qualitative theory of differential equations, 2nd ed., GITTL, Moscow 1949; English transl., Princeton Math. Series, no. 22, Princeton Univ. Press, Princeton, N. J., 1960. MR 10, $612 ; 22$ \# 12258 